\title{
Divergence theorems in path space II: degenerate diffusions
}

\author{
Denis Bell ${ }^{1}$ \\ Department of Mathematics, University of North Florida, \\ 4567 St. Johns Bluff Road South, Jacksonville, FL 32224, U. S. A. \\ email: dbell@unf.edu, phone: 904-620-2653, fax: 904-620-2818.
}

\begin{abstract}
Let $x$ denote an elliptic diffusion process defined on a smooth compact manifold $M$. In a previous work, we introduced a class of vector fields on the path space of $x$ and studied the admissibility of this class of vector fields with respect to the law of $x$. In the present note, we extend this study to the case of degenerate diffusions.
\end{abstract}

\section{Résumé}

Laisser $x$ indique un diffusion elliptique défini sur un lisse compact variété $M$. Dans un travail précédent, nous avons introduit une classe de champs de vecteur sur l'espace de chemin de $x$ et a étudié le admissibilté de cette classe de champs de vecteur par rapport à la loi de $x$. Dans la note présente, nous étendons cette étude au cas de diffusions dégénérées.

${ }^{1}$ Research partially supported by NSF grant DMS-0451194. 
Let $M$ denote a closed compact $d$-dimensional $C^{\infty}$ manifold and $X_{1}, \ldots, X_{n}$ smooth vector fields defined on $M$. Consider the following Stratonovich stochastic differential equation $^{2}(\mathrm{SDE})$ with fixed initial point $o \in M$

$$
d x_{t}=\sum_{i=1}^{n} X_{i}\left(x_{t}\right) \circ d w_{i}, \quad t \in[0, T] .
$$

Let $C_{o}(M)$ denote the space of continuous paths from $[0, T]$ into $M$ originating at $o$.

Definition. A vector field $Z$ on the path space $C_{o}(M)$ is admissible (with respect to the law of $x$ ) if there exists an $L^{1}$ random variable Div $(Z)$ such that the equality

$$
E[(Z \Phi)(x)]=E[\Phi(x) \operatorname{Div}(Z)]
$$

holds for a dense class of smooth functions $\Phi$ on $C_{o}(M)$.

In [1], we developed a method for establishing the admissibility of a class of vector fields $Z$ on $C_{o}(M)$ of the form

$$
Z_{t}=\sum_{i=1}^{n} X_{i}\left(x_{t}\right) h^{i}(t), \quad t \in[0, T]
$$

where $h^{i}:[0, T] \mapsto \mathbf{R}$ are adapted processes, $i=1, \ldots, n$. It was assumed in [1] that the SDE (1) is elliptic, i.e. the vector fields $X_{1}, \ldots, X_{n}$ span $T M$ at all points of $M$. The purpose of this note is to study the admissibility of vector fields of the form (3) in the degenerate case, i.e. when the ellipticity condition fails. This problem has also been treated by Elworthy, Le Jan \& Li using an approach based on filtering (cf. [4, Section 4.1]).

For each $x \in M$, define $E_{x}$ to be the subspace of $T_{x} M$ spanned by the vectors $X_{1}(x), \ldots, X_{n}(x)$. Assume these vector spaces have the same dimension for all $x \in M$, and define $E$ to be the sub-bundle of $T M, E=\cup_{x \in M} E_{x}$. Following Elworthy, Le Jan \& Li ([4]), we Riemannianize $E$ by defining $<.$, . $>$ to be the inner product on each $E_{x}$ induced from the Euclidean space $\mathbf{R}^{n}$ by the map $X(x) \in L\left(\mathbf{R}^{n}, E_{x}\right)$, where $X(x)\left(h_{1}, \ldots, h_{n}\right) \equiv X_{i}(x) h_{i}$. Here, and in the sequel, we assume that whenever an index in a product is repeated, that index is summed on. Further following [4], we define a connection $\nabla$ on $E$, compatible with this metric, by $\nabla_{V} Z=X(x) d_{V}\left(X^{*} Z\right), Z \in \Gamma(E), V \in T_{x} M$, where $d$ represents the usual derivative of the function $x \in M \mapsto X(x)^{*} Z(x) \in \mathbf{R}^{n}$.

We define a collection of 1 -forms $\omega^{j k}, 1 \leq j, k \leq n$ on $M$ by

$$
\omega^{j k}(V)=<\nabla_{X_{j}} X_{k}, V>-<\nabla_{V} X_{j}, X_{k}>-<T\left(X_{j}, V\right), X_{k}>, V \in T M,
$$

where $T$ is the torsion tensor of the connection $\nabla$.

\footnotetext{
${ }^{2}$ The assumption of no drift in equation (1) is purely for notational convenience.
} 
Theorem 1 Suppose that the sub-bundle E satisfies the integrability condition

$$
\operatorname{span}\left\{\left[X_{i}, X_{j}\right](x), 1 \leq i, j \leq n\right\} \subseteq E_{x}, \forall x \in M .
$$

Let $r=\left(r^{1}, \ldots, r^{n}\right)$ be a path in the $n$-dimensional Cameron-Martin space and suppose $h^{1}, \ldots, h^{n}$ are real-valued processes with initial value 0 satisfying the system of $S D E$ 's

$$
d h^{k}=\omega^{j k}(\circ d x) h^{j}+\dot{r}^{k} d t, \quad 1 \leq k \leq n .
$$

Then the vector field $Z$ on $C_{o}(M)$ defined by (3) is admissible.

Sketch of proof. Let $g: C_{0}\left(\mathbf{R}^{n}\right) \mapsto C_{o}(M)$ denote the Itô map $w \mapsto x$ defined by equation (1). Following the approach in [1], we lift $Z$ to the Wiener space via $g$, i.e. we construct a vector field $r$ on $C_{0}\left(\mathbf{R}^{n}\right)$ such that the following diagram commutes

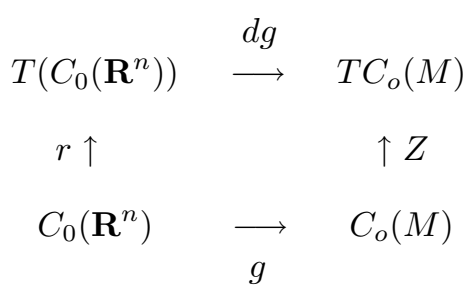

Of course, since the map $g$ is non-differentiable in the classical sense, $d g$ must be interpreted in the extended sense of the Malliavin calculus. The tangent space $T\left(C_{0}\left(R^{n}\right)\right)$ is defined as the space of processes of the form $\int_{o}^{\cdot} h_{s} d s+\int_{0} A_{s} d s$, where $h$ and $A$ are continuous adapted processes with values in $\mathbf{R}^{n}$ and the space of $n \times n$ skew-symmetric matrices $s o(n)$, respectively (this notion of tangent space was inspired by Driver's work [3], see also [5]).

The starting point of the proof is equation (3.6) in [1], which states that $r$ is a lift of $Z$ if and only if the following SDE is satisfied

$$
X_{i}\left(x_{t}\right) \circ d h^{i}=\left[X_{j}, X_{i}\right]\left(x_{t}\right) h^{j} \circ d w_{i}+X_{i}\left(x_{t}\right) d r^{i} .
$$

Now the metric $<., .>$ has the property $V=<V, X_{i}(x)>X_{i}(x)$, for all $V \in E_{x}$. In view of condition (4), we can use this property to solve equation (6) for $d h$ and obtain

$$
d h^{k}=<\left[X_{j}, X_{i}\right]\left(x_{t}\right), X_{k}\left(x_{t}\right)>h^{j} \circ d w_{i}+d r^{k} .
$$

The idea is to now decompose the diffusion coefficient in this equation into a tensorial term in $X_{i}$ and a term that is skew-symmetric in the indices $i$ and $k$. To this end, we define

$$
a_{i k}^{j}(t)=<\nabla_{X_{j}} X_{i}\left(x_{t}\right), X_{k}\left(x_{t}\right)>-<\nabla_{X_{j}} X_{k}\left(x_{t}\right), X_{i}\left(x_{t}\right)>, \quad 1 \leq i, j, k \leq n .
$$


Note that these terms are skew-symmetric in $i$ and $k$. We then have

$$
<\left[X_{j}, X_{i}\right]\left(x_{t}\right), X_{k}\left(x_{t}\right)>=a_{i k}^{j}(t)+\omega_{j k}\left(X_{i}\left(x_{t}\right)\right)
$$

Proceeding as in [1], we write the process $h$ in equation (5) in the form

$$
d h^{k}=<\left[X_{j}, X_{i}\right]\left(x_{t}\right), X_{k}\left(x_{t}\right)>h^{j} \circ d w_{i}+d \tilde{r}^{k}
$$

where

$$
d \tilde{r}^{k}=\dot{r}^{k} d t-a_{i k}^{j}(t) h^{j} \circ d w_{i} .
$$

Thus $\tilde{r}$ is a lift of $Z$. Let $\Phi$ be a test (i.e. smooth cylindrical) function on $C_{o}(M)$. By definition of the lift, we have

$$
E[(Z \Phi)(x)]=E[\tilde{r}(\Phi \circ g)(w)]=E[\Phi(x) \operatorname{Div}(\tilde{r})]
$$

where Div denotes the divergence operator in the classical Wiener space. The form chosen for $\tilde{r}$ ensures that $\operatorname{Div}(\tilde{r})$ exists (cf. Theorems 2.3 and 2.4 in [1]), and the theorem follows.

In general, it is of interest to know if a given vector field on $C_{o}(M)$ admits a lift to the Wiener space. We now show that if Hörmander's condition holds, then the inegrability condition (4) is necessary for the existence of lifts, for almost all vector fields $Z$ on $C_{o}(M)$ of the form (3).

Theorem 2 Suppose condition (4) fails at some point $m \in M$. Define a (proper) subspace $V$ of $\mathbf{R}^{n}$ by

$$
V \equiv\left\{\left(c_{1}, \ldots, c_{n}\right) \in \mathbf{R}^{n} / \operatorname{span}\left\{c_{j}\left[X_{j}, X_{k}\right](m), 1 \leq k \leq n\right\} \subseteq E_{m}\right\} .
$$

Let $h=\left(h^{1}, \ldots, h^{n}\right)$ denote a continuous adapted process in $\mathbf{R}^{n}$ such that $P\left(h\left(t_{0}\right) \notin\right.$ $V)>0$ for some $t_{0} \in(0, T)$. Suppose $X_{1}, \ldots, X_{n}$ satisfy Hörmander's condition everywhere on $M$. Then the vector field $Z$ on $C_{o}(M)$ defined by (3) admits no lift to $C_{0}\left(\mathbf{R}^{n}\right)$ via the Itô map.

Sketch of proof. By hypothesis, there exists $1 \leq k \leq n$ such that $P\left(\left[X_{j}, X_{k}\right](m) h_{t_{0}}^{j} \notin\right.$ $\left.E_{m}\right)>0$. This implies the existence of a a neighborhood $N$ of $m$ on which this condition holds. By a result of Léandre [7, Theorem II.1), $P\left(x_{t_{0}} \in N\right)>0$. In particular, there exists a positive stopping time $\tau$ such that with positive probability

$$
\left[X_{j}, X_{k}\right]\left(x_{t}\right) h_{t}^{j} \notin E_{x_{t}}, \forall t \in\left[t_{0}, t_{0}+\tau\right) .
$$

Now suppose there exists a lift of $Z$ to $C_{0}\left(\mathbf{R}^{n}\right)$. Then equation (6) implies

$$
\left[X_{j}, X_{k}\right]\left(x_{t}\right) h_{t}^{j} \circ d w_{k} \in E_{x_{t}} .
$$


Together, (7) and (8) imply there exists a non-vanishing continuous adapted process $a=\left(a_{1}, \ldots, a_{n}\right)$ such that with positive probability $a_{k}(t) \circ d w_{k}=0, \forall t \in\left[t_{0}, t_{0}+\tau\right)$. However, using the Itô rules $d w_{i} d w_{j}=\delta_{i j} d t, d w_{i} d t=0$, we see this is impossible. This proves that no such lift exists, as claimed.

The following result, which provides a natural setting for Theorem 2 , is easy to verify.

Proposition. Suppose the SDE (1) is degenerate and the vector fields $X_{1}, \ldots, X_{n}$ satisfy Hörmander's condition everywhere on $M$. Then the set of points at which condition (4) fails is dense in $M$.

Remark. The lifting method was originally used by Malliavin [8] to study the hypoellipticity of the differential operator $\sum_{i=1}^{n} X_{i}^{2}$. In this context, it suffices to construct the lift of vector fields on $M$ under the endpoint map $g_{t}: w \mapsto x_{t}$, for fixed $t>0$. Now, it is well-known that when the diffusion (1) is degenerate, Hörmander's condition on $X_{1}, \ldots, X_{n}$ implies the existence of lifts for all smooth vector fields on $M$ under $g_{t}$ (see e.g. [8], [6], [2]). By contrast, Theorem 2 and the Proposition imply that the set of liftable vector fields on the path space of the diffusion is very sparse. The problem of lifting vector fields at the path space level thus has a strikingly different character to that encountered in earlier work on the endpoint problem.

\section{References}

[1] D. Bell, Divergence theorems in path space, J. Funct. Anal. 218 (no. 1), 130 $149,2005$.

[2] D. Bell and S. Mohammed, An extension of Hörmander's theorem for infinitely degenerate second-order operators, Duke Math. J. 78, no. 3: 453-475, 1995.

[3] B. Driver, A Cameron-Martin type quasi-invariance theorem for Brownian motion on a compact manifold. J. Funct. Anal. 109 (1992) 272-376.

[4] K. D Elworthy, Y. Le Jan and X-M Li, On the Geometry of Diffusion Operators and Stochastic Flows, Lecture Notes in Mathematics 1720, Springer-Verlag, 1999.

[5] J. D. S. Jones and R. Léandre, $L^{p}$-Chen forms on loop spaces. Stochastic Analysis (Durham, 1990), 103-162, London Math. Soc. Lecture Note Ser., 167, Cambridge Univ. Press, 1991.

[6] S. Kusuoka and D. Stroock. Applications of the Malliavin calculus II. J. Fac. Sci. Univ. Tokyo 32 (1985) 1-76.

[7] R. Léandre, Appliquations quantitatives et qualitatives du Calcul de Malliavin, Lecture Notes in Mathematics 1322, 109-133, Springer-Verlag, 1988.

[8] P. Malliavin, Stochastic calculus of variations and hypoelliptic operators. Proceedings of the International Conference on Stochastic Differential Equations, Kyoto, 195-263. Kinokuniya and Wiley, 1976. 